\title{
Atividade física e uso de equipamentos entre usuários de duas Academias ao Ar Livre
}

\author{
Physical activity and equipment usage in two fitness zones \\ Bruno Gonçalves Galdino da Costa', Cíntia de la Rocha Freitas², Kelly Samara da Silva²
}

\section{Resumo}

O objetivo do estudo foi comparar características sociodemográficas, percepção de uso dos equipamentos e prática de atividade física moderada a vigorosa (AFMV) entre frequentadores de duas Academias ao Ar Livre (AAL), em Florianópolis (SC), 2013. Entrevista estruturada foi realizada com 217 usuários (58,7\% mulheres), com idade média de 50,2 $( \pm 0,9)$ anos, que estivessem utilizando as AAL durante o período de coleta. Utilizou-se o teste Qui-quadrado para comparar características sociodemográficas, percepção de uso dos equipamentos e AFMV ( $\geq 150$ minutos/semana), entre as AAL. Não foram observadas diferenças estatisticamente significativas entre os usuários das AAL quanto às variáveis sociodemográficas $(p>0,05)$. Quanto ao uso dos equipamentos, $31,3 \%$ dos usuários não sabiam utilizar os aparelhos, $16 \%$ deles relataram sentir desconfortos durante o uso, $23 \%$ relataram ter recebido instrução durante a prática e $69 \%$ afirmaram necessitar de instrução para realizar exercício. Diferenças estatísticas não foram encontradas entre o uso de equipamentos e as AAL ( $\mathrm{p}>0,05)$. Quando analisada a AFMV, percebeu-se que 73\% dos usuários atingiram 150 minutos ou mais, sendo que $19 \%$ deles atingiram esse tempo utilizando apenas as AAL para praticar AFMV. A proporção de prática de AFMV total e nas AAL não diferiu entre os locais estudados ( $\mathrm{p}=0,374$ e $\mathrm{p}=0,495$, respectivamente). Entretanto, usuários da AAL do Córrego Grande relataram maior frequência semanal de ida às AAL $(\mathrm{p}=0,036)$, quando comparados aos usuários de Coqueiros. Conclui-se que os frequentadores das duas AAL apresentam características sociodemográficas, de uso dos equipamentos e de prática de AFMV bastante semelhantes, exceto quanto à frequência de uso das academias.

\section{Palavras-chave}

Atividade física; Exercício; Academias de ginástica; Saúde pública.

\begin{abstract}
The aim of the study was to compare sociodemographic characteristics, perception of the equipment and physical activity levels between users of two Open Fitness Zones (AAL) in Florianopolis (SC). A structured interview was applied with 217 users (58,7\% women) with mean age of $50.2( \pm 0,9)$ years that were using $A A L$ during the study. Chi-squared tests were used to compare sociodemographic variables, perception of the equipment and PA levels between the AAL. No differences were observed between users from different $A A L$ regarding sociodemographic characteristics. As for the perception of the equipment, 31,3\% of the interviewed did not know how to use the equipment, $16 \%$ reported discomforts while using the equipment, $23 \%$ reported having received instruction $\mathrm{du}$ ring practice and $69 \%$ reported the need of instruction to exercise. No statistical differences were found between the perception of the equipment between AAL ( $>>0,05)$. When MVPA was analyzed, we perceived that $73 \%$ of the users achieved 150 minutes or more, and $19 \%$ achieved that goal using only the equipment from $A A L$. The proportions of total MVPA and MVPA in the AAL did not differ between sites ( $p=0.374$ and $p=0.495$, respectively). However, users in Corrego Grande reported a greater weekly frequency of using the AAL ( $p=0.036)$ when compared to users from Coqueiros. Thus concludes that the users from two AAL showed similar sociodemographic profiles, equipment usage variables and had similar physical activity practice and only the weekly frequency of use of the $A A L$ was different between sites.
\end{abstract}

\section{Keywords}

Physical Activity; Exercise; Public Health; Fitness Center

\section{Introdução}

A prática de atividade física $(\mathrm{AF})$ apresenta-se como um fator de proteção para diversos problemas de saú-

1 Núcleo de Pesquisa em Atividade Física e Saúde, Florianópolis, Santa Catarina, Brasil. 2 Universidade Federal de Santa Catarina, Departamento de Educação Física, Florianópolis, Santa Catarina, Brasil. $\mathrm{de}^{1}$, além de promover bem-estar físico e psicossocial ${ }^{2}$ e melhorar a qualidade de vida dos praticantes ${ }^{3}$. Apesar do conhecimento científico acumulado sobre os benefícios da prática regular de $\mathrm{AF}$, o fato de ser fisicamente ativo ainda não é um hábito comum à população mundial ${ }^{4}$. A população brasileira ${ }^{5}$, mesmo com 
a implementação de políticas ${ }^{6}$, planos e programas de promoção da $\mathrm{AF}^{7}$, não tem apresentado um perfil diferente.

A Organização Mundial da Saúde preconiza que adultos saudáveis devem acumular, ao longo da semana, um total de 150 minutos de atividades físicas moderadas a vigorosas (AFMV) para terem benefícios à saúde ${ }^{8}$. Para que essa meta seja atendida tem-se priorizado na Política Nacional de Promoção da Saúde ${ }^{6}$ a ampliação de oportunidades para a AF. Com isso, foram implementados, nos últimos anos, inúmeros projetos comunitários de incentivo à prática de $\mathrm{AF}$, como as Academias da Cidade, em Recife, PE e em Aracajú, SE ${ }^{9,10}$ e, posteriormente, o Programa Academia da Saúde, com estímulo à criação de espaços adequados e profissionais qualificados para atuarem com promoção de saúde e da $\mathrm{AF}^{11}$.

Paralelamente a esses acontecimentos, em diversas cidades brasileiras, dentre elas Florianópolis (SC), surgem as academias ao ar livre (AAL), também chamadas de Academias da Terceira Idade ${ }^{12}$. Outras terminologias têm sido empregadas para referir-se a essas academias, como Academia da Melhor Idade, ou até mesmo, Academia da Saúde (porém, não confundir com Programa Nacional do Ministério da Saúde) para caracterizar tais locais. As AAL tiveram sua origem no projeto Maringá Saudável, em 2006, com propósito de melhorar a saúde dos moradores desta cidade. Essa parceria entre ONGs e empresas privadas resultou em Hortas Comunitárias, promoção de alimentação saudável, "Espaço Saúde", controle de tabagismo e as AAL com o nome Academias da Terceira Idade ${ }^{13}$. A partir de então, as AAL expandiram-se para outros municípios, com sua implantação sendo custeada por órgãos privados, em parceria com as prefeituras locais.

As AAL são equipadas com aparelhos de ginástica, na intenção de melhorar a flexibilidade, mobilidade articular e a capacidade cardiovascular dos usuários ${ }^{12}$. Foram encontrados estudos sobre o perfil de usuários de AAL de Maringá ${ }^{13}$, Curitiba ${ }^{14}$ e Joinville $e^{15}$. Ainda não se tem um panorama das características dos usuários das AAL e se desconhece como esses usuários percebem e utilizam os equipamentos, bem como não foram encontrados registros que mostrem possíveis diferenças entre os praticantes, quando esta modalidade de academia é implantada em parques com maior ou menor infraestrutura. Assim, o objetivo do presente estudo foi comparar características sociodemográficas, a percepção de uso dos equipamentos e a prática de AFMV entre frequentadores de duas Academias ao Ar Livre (AAL) localizadas em dois bairros de Florianópolis (SC).

\section{Métodos}

O presente estudo apresenta delineamento transversal e foi conduzido nos meses de Fevereiro e Março de 2013. Neste período, Florianópolis contava com pouco menos de $30 \mathrm{AAL}$, e com planos para passar de 50, no ano de 2014. As AAL são espaços construídos em parques, praças, espaços públicos e possuem aparelhos para prática de $\mathrm{AF}$, que são utilizados por pessoas de todas as idades ${ }^{12}$.

Para esse estudo, duas AAL foram selecionadas por amostragem intencional, em dois parques de Florianópolis - SC, uma situada em um bairro central da cidade (Córrego Grande) e outra em um bairro continental da cidade (Coqueiros). A escolha dos dois parques ocorreu em função de serem, até o momento da coleta, os únicos locais de Florianópolis com AAL (equipamentos para a prática de AF).

Para conhecimento da infraestrutura dos parques, conduziu-se uma observação direta, onde se pode observar que o parque ecológico municipal João David Ferreira Lima, localizado no bairro Córrego Grande, na região insular de Florianópolis é administrado pela Prefeitura Municipal de Florianópolis. O parque possui 
três trilhas ecológicas, uma AAL (com oito aparelhos), um parquinho com brinquedos para crianças (escorregador, balanços, cordas para escalar, entre outros brinquedos), uma pista de corrida e caminhada de chão batido, com aproximadamente $1,1 \mathrm{~km}$, com aparelhos de barra e prancha abdominal. O parque também conta com espaços amplos de grama que são utilizados para diversas práticas corporais, dois espaços de quadra de areia, de futebol e vôlei, um tanque de areia e uma praça para crianças. Este parque está situado em um ambiente de reserva ecológica, muito arborizado e com animais típicos (aves, mamíferos e répteis, principalmente). O estacionamento deste parque é pequeno, disponibilizando vagas para poucos veículos (em torno de dez). A renda do setor censitário, onde este parque está inserido, é de $\mathrm{R} \$ 1.340 .533,00^{16}$.

O parque de Coqueiros, localizado no bairro de Coqueiros, região continental da cidade, é administrado pela Secretaria do Continente. O parque contém amplos campos de grama utilizados para as mais diversas práticas, como soltar pipa, jogos com bola, slacklines, entre outras. O parque conta com pista para caminhada, corrida e bicicleta, com cerca de 850 metros, duas quadras de futebol de areia, uma de futebol suíço, uma de vôlei de areia e outra de basquetebol. Também estão disponíveis um espaço para a AAL (com sete aparelhos), uma multi estação com barras, paralelas e pranchas abdominais e um parquinho para crianças. O estacionamento do parque oferece vagas para cerca de 800 veículos. A renda do setor censitário no qual este parque está inserido soma cerca de $\mathrm{R} \$ 1.091 .333,00$, um pouco menos do que o observado no bairro Córrego Grande ${ }^{16}$. Em ambos os parques são oferecidas aulas de Yoga e grupos de caminhada gratuita à comunidade.

A amostra foi composta por todos que estivessem utilizando as AAL durante os horários de coleta de dados da pesquisa, que declarassem ser maiores de 18 anos e aceitassem participar voluntariamente da mesma. As coletas de dados foram conduzidas em 2013, nos dias 27 de fevereiro e 03 de março, na AAL de Coqueiros, e nos dias 17 de março e 15 de abril na AAL do Córrego Grande. O espaçamento entre os períodos de coleta é justificado pela necessidade de manter condições climáticas semelhantes nos dias que ocorreram as entrevistas. A coleta foi conduzida durante o turno diurno (de 7:00h às 12:00h; de 14:00h às 17:00h) em ambos os parques, e no turno da noite, de 18:00h às 21:00h, em Coqueiros, e de 18:00h às 19:00h, no Córrego Grande, devido ao horário de funcionamento deste parque.

$\mathrm{O}$ instrumento para coleta de informações foi desenvolvido especificamente para este estudo, no formato de entrevista aplicada, e foi composto por três seções de questões. No primeiro bloco destaca-se as características do participante (idade, sexo, escolaridade, doenças crônicas e lesões recentes); no segundo, a prática de AF habitual (tipos de AF praticadas habitualmente no lazer, frequência e duração das sessões) e, no terceiro bloco, aspectos referentes à utilização das AAL (frequência de utilização, turno mais frequente, duração do tempo de prática no espaço, prática de alongamentos, conhecimento sobre a utilização dos aparelhos presentes, controle do número de séries, repetições, ou tempo em cada aparelho, relato de dores ou desconfortos durante o uso dos aparelhos, relato de instruções de profissionais quanto ao uso dos equipamentos, relato quanto à necessidade de instrução para o uso, e por fim, descrição do tempo de deslocamento da residência ao parque). Detalhes quanto à forma como as variáveis foram perguntadas aos usuários e as categorias de respostas estão descritas no Quadro 1. 
QUADRO 1 - Formato das perguntas com as categorias de resposta que compuseram a entrevista estruturada. Florianópolis, SC, 2013.

\begin{tabular}{l} 
Pergunta (Categorias de resposta) \\
"Quantos anos o(a) Sr(a) tem? (Campo aberto) \\
"O Sr(a) possui diabetes, pressão alta, osteoporose ou alguma outra doença diagnosticada por um médico?" \\
(Diabetes, Hipertensão, Osteoporose, Outro) \\
"O Sr(a) sofreu alguma lesão recentemente? Se sim, em qual parte do corpo?" (Sim ou não; campo aberto) \\
\hline "Até qual ano o Sr(a) estudou?" (Fundamental: incompleto, completo; Médio incompleto, completo, Técnico, \\
Superior incompleto, Completo, Pós-graduação) \\
\hline "O Sr(a) pratica atividade física regularmente? Se sim, quantos dias na semana? (Sim ou não; 0-7 dias) \\
\hline "Quais atividades o Sr(a) pratica com frequência semanal?" (Campo aberto) \\
\hline "Quantos dias por semana o Sr(a) pratica <a atividade citada anteriormente>?" (de 1 a 7 dias por semana) \\
\hline "Quanto tempo dura, em média, uma sessão da <atividade citada anteriormente>?" (Campo aberto) \\
\hline "O Sr(a) frequenta ou já frequentou alguma academia paga?" (Sim ou não) \\
\hline "O Sr(a) utiliza a academia da terceira idade com frequência? Se sim, quantos dias por semana?" (de 1 a 7 dias \\
por semana) \\
\hline "Quanto tempo o Sr(a) passa na academia da terceira idade utilizando os aparelhos?" (Campo aberto) \\
\hline "O Sr(a) faz alongamentos antes de utilizar a academia da terceira idade?" (Sim ou Não) \\
\hline "O Sr(a) sabe utilizar todos os aparelhos da academia da terceira idade?" (Sim ou Não) \\
\hline "O Sr(a) utiliza sempre os mesmos aparelhos?" (Sim ou Não) \\
\hline "O Sr(a) conta o tempo ou o número de repetições em cada aparelho, ou não controla quanto tempo passa em \\
cada um deles?" (Tempo, repetições, não controla) \\
\hline "O Sr(a) já sentiu alguma dor ou desconforto ao utilizar os aparelhos da academia da terceira idade?" (Sim ou \\
Não) \\
\hline "O Sr(a) já recebeu instrução sobre como utilizar a academia da terceira idade?" (Sim ou Não) \\
\hline "Quanto tempo o Sr(a) demora para se locomover de casa até a academia da terceira idade?" (Campo aberto) \\
\hline
\end{tabular}

O instrumento foi submetido à validação de conteúdo (participação de três doutores em Educação Física) e clareza (participação de usuários das AAL), alcançando um índice de 0,9 de validade de conteúdo. Após validação, foi conduzido um estudo piloto realizado em 2012, em uma AAL localizada na cidade de São José (SC), para testar o protocolo da pesquisa.

Quanto à coleta de dados, os usuários foram abordados por um único pesquisador, previamente treinado, à medida que iniciavam os exercícios nos aparelhos. Caso aceitassem participar, o termo de consentimento livre e esclarecido era assinado, e a entrevista, realizada (duração média de 5 minutos).

A variável "idade" foi categorizada em faixas etárias (19-29 anos, 30-39, 4049, 50-59, 60 ou mais anos), para o agrupamento dos respondentes. As variáveis "doenças" e "lesões" foram classificadas em duas categorias (presença: um ou mais casos; ausência: sem nenhum caso). Para a variável "AF", a intensidade das atividades relatadas foi obtida considerando a classificação do equivalente metabólico (MET) igual ou superior a três ${ }^{17}$. Em seguida, foi computado o volume de prática de AFMV, a partir do produto da frequência semanal e duração de cada AF relatada. Posteriormente, foi utilizado o ponto de corte preconizado pela OMS, para o atendimento ou não das recomendações de $\mathrm{AF}^{8}$. Para análise dos dados, utilizou-se a estatística descritiva e aplicou-se o teste Qui-Quadrado para comparar as variáveis investigadas entre as AAL. O programa estatístico utilizado foi SPSS For Windows, versão 15.0. O nível de significância adotado nas análises foi de 5\%.

O projeto foi submetido e aprovado pelo Comitê de Ética em Pesquisas com Seres Humanos da Universidade Federal de Santa Catarina (protocolo número 216.390). 


\section{Resultados}

O número total de usuários convidados foi 223, havendo seis recusas. Dos 217 entrevistados nos dois parques, 147 foram na AAL de Coqueiros, sendo 68 usuários no dia 27 de fevereiro (Quarta-feira) e 79 usuários no dia 03 de março (Domingo), enquanto na AAL do Córrego Grande, foram entrevistados 39 usuários, no dia 17 de março (Domingo), e 31 usuários, no dia 15 de abril (segunda-feira). Houve um maior número de entrevistados nos finais de semana $(n=118)$ do que em dias de semana $(\mathrm{n}=99)$. Quanto às características dos frequentadores, $58,1 \%$ eram mulheres, com média de idade de 50,2 ( $\pm 0,9)$ anos, 53,7\% dos usuários possuíam grau de escolaridade superior, $74,2 \%$ não relataram presença de doenças e $79,3 \%$ reportaram a ausência de lesões. Nenhum desses indicadores diferiu entre os dois locais de coleta $(\mathrm{p}>0,05)$ (Tabela 1$)$. Cerca de um terço dos participantes relatou não saber utilizar os aparelhos das AAL (Coqueiros: 29,9\% e Córrego Grande: 34,3\%, $\mathrm{p}=0,518)$ e uma proporção semelhante relatou utilizar sempre os mesmos aparelhos (Coqueiros: 30,6\% e Córrego Grande: 25,7\%, p=0,276) (Tabela 1).

TABELA 1 - Característica dos frequentadores e comparação das variáveis de estudo por localização dos parques. Florianópolis, SC. 2013.

\begin{tabular}{|c|c|c|c|c|c|c|c|}
\hline \multicolumn{8}{|c|}{ Localização dos Parques } \\
\hline \multirow[t]{2}{*}{ Variáveis } & \multicolumn{2}{|c|}{$\begin{array}{l}\text { Coqueiros } \\
(n=147)\end{array}$} & \multicolumn{2}{|c|}{$\begin{array}{l}\text { Córrego } \\
(n=70)\end{array}$} & \multirow{2}{*}{$p^{*}$} & \multicolumn{2}{|c|}{$\begin{array}{c}\text { Total } \\
(n=217)\end{array}$} \\
\hline & $n$ & $\%$ & $\mathrm{n}$ & $\%$ & & $n$ & $\%$ \\
\hline \multicolumn{8}{|l|}{ Pessoais } \\
\hline Sexo & & & & & 0,690 & & \\
\hline Masculino & 63 & 42,9 & 28 & 40,0 & & 91 & 41,9 \\
\hline Feminino & 84 & 57,1 & 42 & 60,0 & & 126 & 58,1 \\
\hline Idade (anos) & & & & & 0,117 & & \\
\hline $19-29$ & 9 & 6,2 & 10 & 14,3 & & 19 & 8,8 \\
\hline $30-39$ & 20 & 13,8 & 7 & 10 & & 27 & 12,6 \\
\hline $40-49$ & 38 & 26,2 & 12 & 17,1 & & 50 & 23,3 \\
\hline $50-59$ & 46 & 31,7 & 21 & 30,0 & & 67 & 31,1 \\
\hline$\geq 60$ & 32 & 22,1 & 20 & 28,6 & & 52 & 24,2 \\
\hline Escolaridade & & & & & 0,375 & & \\
\hline Fundamental & 24 & 16,6 & 7 & 10,1 & & 31 & 14,5 \\
\hline Médio & 43 & 29,7 & 25 & 36,2 & & 68 & 31,8 \\
\hline Superior & 78 & 53,7 & 37 & 53,7 & & 115 & 53,7 \\
\hline Presença de Doenças & & & & & 0,493 & & \\
\hline Nenhuma & 107 & 72,8 & 54 & 77,1 & & 161 & 74,2 \\
\hline 1 ou mais & 40 & 27,2 & 16 & 22,9 & & 56 & 25,8 \\
\hline Presença de Lesões & & & & & 0,595 & & \\
\hline Nenhuma & 118 & 80,3 & 54 & 77,1 & & 172 & 79,3 \\
\hline 1 ou mais & 29 & 13,7 & 16 & 22,9 & & 45 & 20,7 \\
\hline \multicolumn{8}{|l|}{ Uso dos equipamentos } \\
\hline Sabe utilizá-los & & & & & 0,518 & & \\
\hline Sim & 103 & 70,1 & 46 & 65,7 & & 149 & 68,7 \\
\hline Não & 44 & 29,9 & 24 & 34,3 & & 68 & 31,3 \\
\hline Utiliza sempre os mesmos & & & & & 0,276 & & \\
\hline Sim & 102 & 69,4 & 51 & 73,9 & & 153 & 70,8 \\
\hline Não & 45 & 30,6 & 18 & 26,1 & & 63 & 29,2 \\
\hline Como faz o controle do uso & & & & & 0,054 & & \\
\hline
\end{tabular}

Continua... 
... continua

\begin{tabular}{lccccccc}
\hline $\begin{array}{l}\text { Controla (tempo ou } \\
\text { repetiçães) }\end{array}$ & 70 & 47,6 & 42 & 62,9 & & 112 & 52,1 \\
\hline Não faz controle & 77 & 52,4 & 26 & 37,1 & & 103 & 47,9 \\
\hline $\begin{array}{l}\text { Já sentiu dor ou desconforto } \\
\text { Sim }\end{array}$ & 26 & 17,8 & 9 & 12,9 & & 35 & 16,2 \\
\hline Não & 120 & 82,2 & 61 & 87,1 & & 181 & 83,8 \\
\hline $\begin{array}{l}\text { Já recebeu instrução para } \\
\text { o uso }\end{array}$ & & & & & 0,965 & & \\
\hline Sim & 34 & 23,1 & 16 & 22,9 & & 50 & 23,0 \\
\hline Não & 113 & 76,9 & 54 & 77,1 & & 167 & 77,0 \\
\hline $\begin{array}{l}\text { Sente necessidade de } \\
\text { instruções }\end{array}$ & & & & & 0,346 & & \\
\hline Sim & 97 & 67,4 & 51 & 72,9 & & 148 & 69,2 \\
\hline Não & 47 & 32,6 & 19 & 27,1 & & 66 & 30,8 \\
\hline
\end{tabular}

*Teste qui-quadrado de heterogeneidade. **Teste qui-quadrado de tendência.

TABELA 2 - Atividade física dos frequentadores e comparação das variáveis de estudo por localização dos parques. Florianópolis, SC. 2013.

\begin{tabular}{|c|c|c|c|c|c|c|c|}
\hline \multirow{3}{*}{ Variáveis } & \multicolumn{5}{|c|}{ Localização dos Parques } & & \\
\hline & \multicolumn{2}{|c|}{$\begin{array}{l}\text { Coqueiros } \\
(n=147)\end{array}$} & \multicolumn{2}{|c|}{$\begin{array}{l}\text { Córrego } \\
(n=70)\end{array}$} & \multirow{2}{*}{$p^{*}$} & \multicolumn{2}{|c|}{$\begin{array}{c}\text { Total } \\
(n=217)\end{array}$} \\
\hline & $n$ & $\%$ & $n$ & $\%$ & & $n$ & $\%$ \\
\hline Faz Alongamentos & & & & & 0,692 & & \\
\hline Sim & 105 & 71,9 & 48 & 68,6 & & 153 & 70,8 \\
\hline Não & 41 & 28,1 & 22 & 31,4 & & 63 & 29,2 \\
\hline Frequência de uso de AAL & & & & & $0,036^{* *}$ & & \\
\hline Irregular & 40 & 27,2 & 7 & 10,0 & & 47 & 21,7 \\
\hline $1-2$ dias & 14 & 9,5 & 10 & 14,3 & & 24 & 11,0 \\
\hline 3-4 dias & 36 & 24,5 & 21 & 30 & & 57 & 26,3 \\
\hline 5-7 dias & 57 & 38,8 & 32 & 45,7 & & 89 & 41,0 \\
\hline Volume AFMV (sem AAL) & & & & & $0,038^{*}$ & & \\
\hline$<150 \mathrm{~min} / \mathrm{semana}$ & 68 & 46,3 & 22 & 31,4 & & 90 & 41,5 \\
\hline$\geq 150 \mathrm{~min} / \mathrm{semana}$ & 79 & 53,7 & 48 & 68,6 & & 127 & 58,5 \\
\hline Volume AFMV (apenas AAL) & & & & & 0,495 & & \\
\hline$<150 \mathrm{~min} / \mathrm{semana}$ & 116 & 78,9 & 58 & 82,9 & & 174 & 80,2 \\
\hline$\geq 150 \mathrm{~min} / \mathrm{semana}$ & 31 & 21,1 & 12 & 17,1 & & 43 & 19,8 \\
\hline Volume AFMV Total & & & & & 0,374 & & \\
\hline$<150 \mathrm{~min} / \mathrm{semana}$ & 42 & 28,6 & 16 & 22,8 & & 58 & 26,8 \\
\hline$\geq 150 \mathrm{~min} / \mathrm{semana}$ & 105 & 71,4 & 54 & 77,2 & & 159 & 73,2 \\
\hline
\end{tabular}

*Teste qui-quadrado de heterogeneidade. ${ }^{* *}$ Teste qui-quadrado de tendência.

Em relação à utilização dos aparelhos nas AAL, 47,9\% dos respondentes relataram não fazer controle do uso dos equipamentos, sem diferenças estatísticas ( $p>0,05)$ entre as AAL. Do total, 16,2\% dos usuários relataram ter sentido dor ou desconforto ao utilizar os aparelhos das AAL, 77\% nunca receberam instrução para utilizar os aparelhos nas AAL e 69,2\% relataram sentir necessidade de instrução para melhor utilizar os equipamentos. Nesses itens, não houve diferenças significativas entre os locais avaliados $(\mathrm{p}>0,05)$ (Tabela 1$)$.

Vinte e três por cento dos entrevistados relataram não fazer alongamento antes de iniciar AF nas AAL, sem diferenças estatísticas entre os locais ( $p>0,05)$. A maioria 
dos usuários (41,0\%) relatou utilizar as AAL 5 a 7 dias por semana, com maior frequência semanal entre os usuários da AAL do parque do Córrego Grande, comparados aos usuários do parque de Coqueiros $(\mathrm{p}=0,036)$ (Tabela 2$)$.

Com relação à $\mathrm{AFMV}, 73,2 \%$ dos frequentadores alcançaram 150 minutos semanais, sendo que 19,8\% alcançaram essa duração praticando AF apenas nas AAL. Com relação à localização dos parques, não foram encontradas diferenças estatísticas significantes quanto ao atendimento às recomendações $(p>0,05)$ (Tabela 2).

\section{Discussão}

O diferencial desse estudo em relação a outros publicados nesta área é que se procurou investigar a característica de usuários que utilizam os aparelhos disponíveis nas academias ao ar livre, confrontando duas instalações localizadas em parques distintos e bastante frequentados na cidade de Florianópolis, SC. As AAL nestes parques oferecem uma oportunidade para os frequentadores serem fisicamente ativos no seu tempo de lazer, em um espaço natural e gratuito para a prática, podendo ser um palco para intervenções e programas comunitários que promovam AF por meio da prática regular de exercício físico.

O presente estudo apresenta limitações em relação ao número de visitas em cada espaço (somente duas visitas), porém houve a preocupação em analisar um dia da semana e outro do final de semana, com condições climáticas semelhantes (dias ensolarados). A diferença no número de respondentes entre os espaços dificulta o uso de análise estatística inferencial mais complexa, no entanto, associações preliminares foram encontradas e modelos ajustados poderão ser testados em futuros estudos. Embora se reconheça a importância de caracterizar e registrar as recusas, esse procedimento não foi adotado durante a coleta, o que dificulta explorar as características dos não respondentes. No entanto, somente foram registradas seis recusas.

Diferentemente dos resultados encontrados neste estudo, outras investigações mostraram que mulheres se engajaram mais do que homens na utilização destes espaços em outras cidades ${ }^{13,14,18}$. A média de idade encontrada entre usuários de academias semelhantes de Joinville foi de $59 \operatorname{anos}^{18}$. Apesar de neste estudo a utilização das AAL não diferir entre variáveis sociodemográficas, foram encontradas maiores proporções de mulheres, usuários com 50-59 anos e com nível de escolaridade superior, conforme estudos previamente citados. As diferenças encontradas entre os estudos podem ser parcialmente explicadas por questões metodológicas, principalmente, a faixa etária, pois o estudo de Carvalho ${ }^{18}$ apenas entrevistou usuários com idades superiores a quarenta anos.

O número de participantes que relatou ter alguma doença ou lesão no presente estudo foi menor do que os 51,2\% dos usuários das academias de Joinville, que relataram ter doenças crônicas ${ }^{18}$. O estudo de Salin ${ }^{14}$, também em Joinville, mostrou que $72,2 \%$ dos idosos entrevistados também relataram alguma doença, porém, como esse estudo foi apenas com idosos, isso pode explicar a maior prevalência de doenças nesse grupo, quando comparado com uma amostra que contém participantes não idosos, e com menor risco para desfechos como hipertensão, osteoporose e diabetes.

Em nosso estudo, foi verificada baixa proporção de participantes que relataram ter recebido instruções sobre a utilização das AAL e elevada proporção daqueles que relataram a necessidade de receber tais instruções. Sabe-se que a falta de controle dos indicadores de exercício (como número de repetições e intensidade por exemplo) no uso dos aparelhos, bem como a utilização sempre dos mesmos 
aparelhos durante as sessões podem ter um impacto no volume de treino, e, consequentemente, influência no gradiente dos benefícios relacionados à prática ${ }^{19}$. Aliado a isso, a falta de confiança ou até mesmo a insegurança na utilização do aparelho pode afetar diretamente não só os indicadores de prática ${ }^{19}$ como a auto eficácia para fazer exercício físico. Um outro elemento importante, é a questão postural no uso do equipamento durante a prática, aumentando a chance de lesões no sistema músculoesquelético ${ }^{20}$.

Um total de 74\% dos idosos frequentadores de AAL de Joinville sugeriram que profissionais de Educação Física estivessem presentes para atender aos grupos, mostrando que essa necessidade é percebida em outros ambientes públicos para a prática de $\mathrm{AF}^{14}$. Possivelmente, a presença desse profissional poderia potencializar a utilização desses espaços, ampliar o número de usuários, melhorar a orientação e execução dos movimentos, e contribuir diretamente para que mais usuários pudessem atingir o volume de AF preconizado e serem inseridos em locais com prática de $\mathrm{AF}$ orientada.

Poucos participantes relataram sentir ou já ter sentido dor ou desconforto utilizando os aparelhos das AAL. Apesar disso, a presença desse sintoma e o desconhecimento em relação a causa da dor é preocupante, pois o exercício físico realizado de forma equivocada ou com postura inadequada pode oferecer riscos de lesões osteoarticulares e musculares ao praticante. No estudo de Oliveira et al. ${ }^{21}$, os usuários relataram sentir mais desconforto do que o encontrado neste estudo, e uma explicação levantada pelos autores é a impossibilidade de ajustes dos equipamentos aos diferentes tamanhos corporais. Tal situação pode representar uma barreira para o uso dos equipamentos, assim como uma avaliação não tão positiva da prática, quando registros de dores e desconfortos são relatados. Nesse sentido, recomendam-se mais estudos sobre queixas e implicações do uso dos equipamentos disponibilizados nesses locais.

Com relação à prática de alongamentos antes das sessões de exercício, não há um corpo de evidências consensual quanto ao seu efeito protetor de lesões durante a prática ${ }^{22}$. No entanto, sabe-se que, em adultos de meia-idade e idosos, o grau de capacidade funcional e, especificamente, limitações articulares, parecem favorecer ou dificultar a execução de alguns movimentos ${ }^{23}$. Desse modo, sugere-se incentivar a execução de exercícios específicos de alongamento antes e após as sessões, para contribuir com um possível ganho de mobilidade funcional, e talvez, prevenir danos osteoarticulares e musculares na faixa etária investigada.

No presente estudo, a maioria dos participantes atende às recomendações de $\mathrm{AF}$, com uma proporção considerável do tempo advindo das AAL. Souza et al. ${ }^{13}$ também encontraram resultados similares, ao indicarem que aproximadamente $72 \%$ dos usuários das AAL de Curitiba atingiam 150 minutos de AF por semana. Esse achado destaca a importância das AAL como um espaço para que os usuários pratiquem AF regularmente, a fim de obterem benefícios e proteção contra doenças crônico-degenerativas. Uma possibilidade não explorada nesse estudo pode ser encontrada nos achados de Souza et al. ${ }^{13}$, Salin ${ }^{14}$ e Esteves et al. ${ }^{15}$, em que o apoio social, a companhia e a formação de grupos pode influenciar positivamente o padrão de utilização de espaços como as AAL, com contribuição para a saúde desses usuários, à medida que praticam mais exercícios dentro desses grupos.

A inserção de profissionais de Educação Física nesses locais parece se apresentar como uma estratégia favorável para suprir a necessidade de instruções relatadas pelos frequentadores, minimizar os relatos de dores e desconfortos durante o uso de equipamentos, e contribuir com a possibilidade de aumentar o volume de 
prática por parte dos usuários. Nos Estados Unidos, os espaços mais procurados por usuários em parques são aqueles que oferecem atividades organizadas ou supervisionadas $^{28}$. Em Recife-PE, pesquisadores observaram que AF orientada por um profissional foi efetiva em atrair e aumentar o engajamento do público em exercícios físicos $^{29}$. Especificamente em Florianópolis, os profissionais de Educação Física, que atuam na Equipe de Saúde da Família no Sistema Único de Saúde, são responsáveis por diversas ações e grupos de atividades estruturadas a partir da demanda dos Centros de Saúde ${ }^{30}$. No entanto, considerando a área de cobertura, ainda há um número restrito de profissionais atuando nesses espaços.

Por fim, conclui-se que as variáveis investigadas não diferiram entre os locais estudados, exceto a frequência semanal de ida à AAL que foi maior no parque do Córrego Grande, em relação ao de Coqueiros. Informações adicionais mostraram que sete em cada dez usuários destes espaços atingem às recomendações de $\mathrm{AF}$, um quarto deles relatou não saber usar os equipamentos e mais de dois terços nunca receberam instruções de uso.

Pesquisas futuras poderiam investigar a totalidade dos parques existentes em Florianópolis, independentemente de possuir AAL, a fim de obter uma informação global do perfil de usuários e das dificuldades relatadas por eles. Tais informações forneceriam mais detalhes sobre aspectos que precisam ser melhorados, como facilitadores e entraves para o uso dos espaços e de equipamentos existentes. Também é importante avançar na combinação de medidas subjetivas e objetivas, utilizando instrumentos objetivos para medir a atividade física e para avaliar parques e outros espaços existentes na cidade para a prática de AF, e ainda aspectos biomecânicos, a quantidade e a diversidade dos aparelhos.

\section{Referências}

1. Kaplan GA, Strawbridge WJ, Cohen RD, Hungerford LR. Natural history of leisuretime physical activity and its correlates: associations with mortality from all causes and cardiovascular disease over 28 years. Am J Epidemiol. 1996 ;144(8):793-7.

2. Penedo FJ, Dahn JR. Exercise and well-being: a review of mental and physical health benefits associated with physical activity. Curr Opin Psychiatr. 2005;18(2):189-93.

3. Gill DL, Hammond CC, Reifsteck EJ, Jehu CM, Williams RA, Adams MM, et al. Physical activity and quality of life. J Prev Med Public Health. 2013;46(Suppl 1):S28-S34.

4. Waxman A. Prevention of chronic diseases: WHO global strategy on diet, physical activity and health. Food Nutr Bull. 2003;24(3).

5. Ministério da Saúde. Vigitel Brasil 2012: vigilância de fatores de risco e proteção para doenças crônico por inquérito telefônico. Brasília, DF. 2013.

6. Ministério da Saúde. Política nacional de promoção da saúde. Brasília, DF. 2010.

7. Malta DC, Castro AMD, Gosch CS, Cruz DKA, Bressan A, Nogueira JD, et al. A política nacional de promoção da saúde e a agenda da atividade física no contexto do SUS. Epidemiol Serv Saúde. 2009;18(1).

8. WHO. Global recommendations on physical activity for health. 2010.

9. Hallal PC, Tenório MCM, Tassitano RM, Reis RS, Carvalho YM, Cruz DKA, et al. Avaliação do programa de promoção da ativiade física academia da cidade de Recife, Pernambuco, Brasil: percepções de usuários e não-usuários. Cad Saúde Pública. 2010;26(1):70-8.

10. Mendonça BCDA, Toscano JJDO, Oliveira ACCD. Do diagnóstico à ação: experiências em promoção da atividade física programa academia da cidade Aracaju: promovendo saúde por meio da atividade física. Rev Bras Ativ Fís Saúde. 2009;14(3):211-6.

11. Brasil, Ministério da Saúde. Portaria no 719, de 07 de abril de 2011. Diário Oficial da União. 2011.

12. Palácios AROP, Salineiro APDSB, Nardi ACF, Rosaneli CF. A concepção do Programa Maringá Saudável, suas ações e resultados. Divulg Saúde Debate. 2009;44:34-40. 
13. Esteves JVDC, Andreato LV, Moraes SMFd, Prati ARC. Estilo de vida de praticantes de atividades físicas em academias da terceira idade de Maringá - PR. Conexões. 2010;8(1).

14. Souza CAD, Fermino RC, Añez CRR, Reis RC. Perfil dos frequentadores e padrão de uso das academias ao ar livre em bairros de baixa e alta renda de Curitiba-PR. Rev Bras Ativ Fís Saúde. 2014;19(1):86-97.

15. Salin MDS. Espaços públicos para a prática de atividade física: o caso das academias da melhor idade de Joinville-SC [Tesde de Doutorado]. Florianópolis: Universidade Estadual de Santa Catarina; 2013.

16. Institutuo Brasileiro de Geografia Estatística. Pesquisa de orçamentos familiares 2008-2009 Antropometria e estado nutricional de crianças, adolescentes e adultos no Brasil. 2010.

17. Ainsworth BE, Haskell WL, Herrmann SD, Meckes N, Bassett DR, Tudor-Locke C, et al. 2011 Compendium of Physical Activities: a second update of codes and MET values. Med Sci Sports Exerc. 2011;43(8):1575-81.

18. Carvalho KBBV. Perfil Socioeconômico e qualidade de vida em usuários do programa academia da melhor idade no município de Joinville-SC [Dissertação de Mestrado]. Joinville:Universidade da Região de Joinville; 2011.

19. Garber CE, Blissmer B, Deschenes MR, Franklin BA, Lamonte MJ, Lee IM, et al. American College of Sports Medicine position stand. Quantity and quality of exercise for developing and maintaining cardiorespiratory, musculoskeletal, and neuromotor fitness in apparently healthy adults: guidance for prescribing exercise. Med Sci Sports Exerc. 2011;43(7):1334-59.

20. Kendall FP. Músculos: provas e funções, com postura e dor. 2 ed. Barueri: Manole; 2007.

21. Oliveira STD, Paschorelli LC, Okimoto MLLR, Carvalho ML. Design universal e acessibilidade: análise ergonômica de equipamentos de ginástica em espaços públicos. II Conferência Internacional de Integração Design, Engenharia e Gestão para a inovação; Florianópolis, SC, Brasil, 2012.

22. Di Alencar TAM, Matias KFDS. Princípios fisiológicos do aquecimento e alongamento muscular na atividade esportiva. Rev Bras Med Esporte. 2010;16:230-4.

23. Beissner KL, Collins JE, Holmes H. Muscle force and range of motion as predictors of function in older adults. Phys Ther. 2000;80(6):556-63.

24. Livingstone MB, Robson PJ, McCarthy S, Kiely M, Harrington K, Browne P, et al. Physical activity patterns in a nationally representative sample of adults in Ireland. Public Health Nutr. 2001;4(5a):1107-16.

25. Livingstone MB, Robson PJ, Wallace JM, McKinley MC. How active are we? Levels of routine physical activity in children and adults. Proc Nutr Soc. 2003;62(3):681-701.

26. Crespo CJ, Ainsworth BE, Keteyian SJ, Heath GW, Smit E. Prevalence of physical inactivity and its relation to social class in U.S. adults: results from the Third National Health and Nutrition Examination Survey, 1988-1994. Med Sci Sports Exerc. 1999;31(12):1821-7.

27. Jones DA, Ainsworth BE, Croft JB, Macera CA, Lloyd EE, Yusuf HR. Moderate leisuretime physical activity: who is meeting the public health recommendations? A national cross-sectional study. Arch Fam Med. 1998;7(3):285-9.

28. Cohen DA, Lapham S, Evenson KR, Williamson S, Golinelli D, Ward P, et al. Use of neighbourhood parks: does socio-economic status matter? A four-city study. Public Health. 2013;127(4):325-32.

29. Parra DC, McKenzie TL, Ribeiro IC, Ferreira Hino AA, Dreisinger M, Coniglio K, et al. Assessing physical activity in public parks in Brazil using systematic observation. Am J Public Health. 2010;100(8):1420-6.

30. Prefeitura Municipal de Florianópolis. Registro de grupos sob responsabilidade dos profissionais de educação física no NASF. Secretaria Municipal de Saúde. 2014.

ENDEREÇO PARA

CORRESPONDÊNCIA

BRUNO GONÇALVES

GALDINO DA COSTA

brunoggdacosta@gmail.com
Rua Leo A. Petry, número 191, Apartamento 302,

São José, Santa Catarina, Brasil. CEP: 88103-610

(48) 3034-3933/ (48)9928-5288 